\title{
Alternative frameworks amongst University of Plymouth Astronomy Students
}

\author{
By Mike P.V. Broughton
}

Centre for Teaching Mathematics, University of Plymouth, Plymouth, PL4 8AA

\section{Introduction}

In recent years much research into conceptual understanding of science has been carried out. Oddly, Astronomy (one of the smallest sciences in terms of pupil numbers) is possibly one of the most widely studied subjects, with numerous papers being produced revealing the intuitive ideas of (usually) young school children. Within these papers it is generally recognised that if students cannot assimilate the fundamental concepts of a subject, then their own initial frameworks are altered accordingly, producing mis-conceptions.

Much of this research into pre/mis-conceptions, alternative frameworks etc, has been concerned with the knowledge of gravity or the shape of the Earth, the Sun and other such bodies. Another area heavily researched is that of phases/eclipses, and how the young children of today perceive these phenomena.

The research presented here takes the findings from earlier papers and extends it by assessing astronomy students at the University of Plymouth. The experiment probed two areas, the phases and eclipses of the moon and Sun and the ability of students to de-centre.

\section{Previous Studies}

It has been known for many years now that children usually start to think of the Earth as flat (Vosniadou et al (1989)), with age usually removing or adjusting initial frameworks. This may be demonstrated by assuming we have two children, $A$ and $B$, which both hold the notion of a flat Earth. From the flat Earth model, child A may 'leap' to the concept of a spherical Earth straight away; the child's flat Earth conceptions have been removed and replaced with a model which the child is able to associate with 'space' and thus a spherical Earth. Child B may only have an adjustment of models however, and would 'add' to its current framework the notion of a spherical Earth. This adjustment has two consequences. The original model of a flat Earth is reinforced in the child's memory, not because the child has been told that flat Earths exist, but because the child has not been told. The second, and more damagingt in terms of knowledge is that the child now falls into the category of 'Dual Earths'.

A 'Dual Earth' situation is one in which the flat Earth is the object which Humans, and all life, live on. The second Earth, a spherical body, is floating in the 'sky' and is usually unobservable by people on the ground. The only way in which we may observe this second Earth is by going 'up'. This happens to be true; the only way we can view the whole Earth is by being in space. Pictures which have helped cause the child to transfer between notions are all of the Earth taken from space, and since it is not mentioned that this is the one and only Earth, the child constructs an additional branch to the current model enabling it to again fit with observable data.

It is apparent in all research that the acquisition of notions, culminating in the correct scientific view, is gained with age. The age spread is typically from about 8 years (still in flat Earth mode) to 15 years old (Correct notion). Mali and Howe (1979) and other researchers have shown that not only does age play a part, but also the culture in which the child is brought up. The results of an experiment conducted by Mali and Howe showed that children in America were approximately one year ahead of their neighbours in Nepal, even with similar schooling. (Approximately is

† The term damaging is used because the model allows the child to tackle quite complex astronomical events and surface with the correct answer, such as phases, whilst holding an incorrect model. 
used because at 15 years old, the end of the study, a large proportion of Nepali children still believed in a flat Earth, which Mali and Howe linked to local religion).

When a child has reached the stage of understanding space and approximate spatial awareness, researchers have switched their main focus to that of how the Solar System moves. Through objects such as polystyrene spheres and balls, researchers ask the subjects to animate the Solar System and roughly indicate what orbits what.

\section{Advancement of previous surveys}

Not much research has been carried out on students after they have left school, although this is probably due to the subject of Astronomy being small and normally only offered at University level. University Astronomy is not concerned with the shape of the Earth, the Moon's orbit (although it does normally cover phases and eclipses) and how our Solar System works, but is aimed more at mathematical and historical events, such as Dopper shift, relativity and Galileo's role in the advancement of science. This assumes that the students who take the subject have a firm grasp of the 'basic' astronomical events.

The University of Plymouth, and its predecessors, have taught astronomy for $100+$ years and it was decided that the students who participate in the course should be tested for their understanding of these 'basic' events. The course is a three year minor pathway on a honours degree, with approximately 106 students in total, although around $80 \%$ of these students are in the first year.

All three years were to participate in the same questionnaire survey which was designed to test for lunar phases/eclipses and also for their de-centering ability. To test the students' phases notions, three questions were presented in the form of a pictorial multiple choice. Each question featured a picture of a lunar phase, with six possible answers each showing a different configuration of the Earth, Sun and Moon.

Two eclipse questions were asked in a format identical to the previous question, each requiring knowledge of lunar and solar eclipses respectively.

The last two questions were designed to test for de-centering ability directly. The first was an invented countryside map with a small village in the middle. Given two views the student was required to place him/her-self on the map. This is a concept which most students are familiar with and so it was expected that the positive results of this question were to be high.

The second de-centering question was similar in style and approach as to the map question, with two views and a map being provided. The map was however a chart of the Solar System at a given time and date, with the two views of various planets. The student was to use this information to locate themselves within the Solar System.

The initial de-centering problem is very similar to those employed by Piaget (1929) in the three mountain problem. Some of the barriers which Piaget faced were overcome by use of the printed questionnaire rather than one-to-one questioning, and by the benefit of using older students who do not require prompting in any way. The second question is again similar but on grounds which students are not familiar with. In terms of method, both should be identical and thus a student who completes the first question correctly should also complete the second equally well.

\section{Results from the questionnaire}

The questionnaire was administered during September 1995, with all students taking the question paper within 3 days of each other. In this paper, the actual results are not going to be studied or analysed in any great detail but rather the relevant comments the students placed in the space at the end of each question for justification and some reflections from the one-to-one interviews.

Questions one through three were asking for answers to problems involving the phases of the moon. Questions four and five asked about lunar and solar eclipses and the last two were 'map' 
locating exercises. The percentage of correct responses to each question and a brief outline of the written responses follow.

Question 1: Link a picture of a thin new moon to its orbital position

It was evident early in the marking that the students were answering correctly yet were giving the wrong reasons for the event. The scores for years 1,2 and 3 are $44 \%, 50 \%$ and $88 \%$.

Question 2: Link a picture of a 1st quarter moon to its orbital position

Scores for year 1,2 and 3 were $75 \%, 75 \%$ and $88 \%$. This question often raised the response that it had to be 'that one' because half of the moon was visible which implied that a $90^{\circ}$ angle must be present.

Question 3: Link a picture of a moon just after full to its orbital position

Scores for years 1,2 and 3 were $46 \%, 62 \%$ and $88 \%$. It should be noted that the third year results are slightly tainted by one person who got every phase question incorrect. Also, the candidate was the only student who justified the correct reasoning yet the wrong picture.

Notes on the phases question

Following is a table showing a summary of the justifications of the students and whether the student got the question correct. (Remarks that were repeated frequently are indicated by an asterisk).

Table 1 - Student Justifications

$\begin{array}{lcl}\text { Justification } & \text { Years } & \text { Solution } \\ \text { The Moon blocks the light } & 1 & \text { Correct } \\ \text { Earth obstructs light } & 1 & \text { Correct } \\ \text { Earth silhouettes moon* } & 1,2 & \text { Incorrect } \\ \text { Shadow of Earth obstructs } & 2 & \text { Incorrect } \\ \text { Earth blocks light* } & 1,2,3 & \text { Incorrect } \\ \text { Earth casts shadow } & 2 & \text { Correct } \\ \text { Only part of moon lit* } & 1,2 & \text { Correct } \\ \text { Light side points away from Sun (!) } & 1 & \text { Incorrect } \\ \text { Moon is at same distance as Sun } & 1 & \text { Correct }\end{array}$

The Table shows an alarming trend which was seen throughout the questionnaire. A number of correct solutions were supplied, but the justification was often widely incorrect, a situation which is commonly termed dual-perspective (Berry et al 1991). Table 1 was created mostly from the first years' answers as years 2 and 3 were not as co-operative and often left the justification blank. Of the nine 2nd years, 4 answered with mis-conceptions such as the Earth casting a shadow. One third year remarked that the closer the moon is to the Sun, the brighter and thus more 'visible' it becomes.

The dual-perspective may be equated with a framework similar to that of the Dual Earth model held by children and mentioned earlier in this paper. The concept of the Earth- MoonSun system and what phases look like when is 'programmed' in, but the underlying conception of a flat Earth style framework exists in memory.

Question 4: Match a picture of a Lunar eclipse with its orbital position

Score for years 1,2 and 3 were $53 \%, 38 \%$ and $78 \%$ respectively.

Question 5 : Match a picture of a Solar eclipse with its orbital position

Score for years 1,2 and 3 were $75 \%, 38 \%, 78 \%$. The low score for this and the question before are due to reversals in both the answer and justification; i.e. a solar eclipse became a lunar eclipse and vice versa. The high percentage of correct answers in year one was also a surprise 


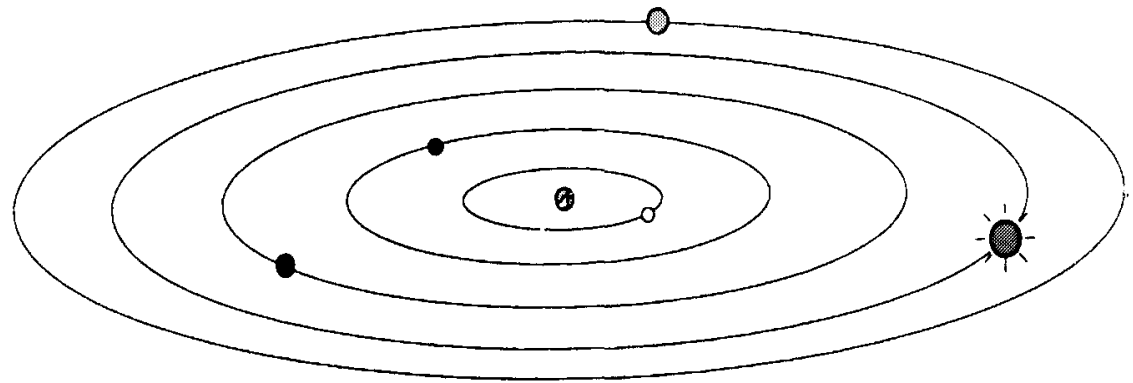

FIGURE 1.

when compared to the previous phase results and may be attributed to the amount of media coverage eclipses obtain.

Question 6 : Locate oneself on the map provided given the views due north and south This question was, unsurprisingly, answered very well by all years with scores of $95 \%, 100 \%$, $100 \%$ for years 1,2 and 3 . The general opinion of this question was that they (students) could not find a reason for asking it.

Question 7: Locate oneself on the starchart provided: two views given, $180^{\circ}$ apart Year one did not like this question at all with $36 \%$ of the students not answering and of those that did, $35 \%$ got it correct. All second and third years answered the question with scores of $38 \%$ and $44 \%$.

\section{Conceptions of our Solar System}

It quickly became apparent that the question on position finding in the last de- centering question was posing quite interesting problems. As an extension to the question paper, one-toone interviews were employed which discovered that the students at the University of Plymouth have pre-conceptions about the Solar System; some of which may only be described as preCopernican.

Following are the results of the extension study. The results, which also included drawings by the students, were able to be classified into five notions. Notion five is the most advanced and is the current scientific view of the Solar System.

Figure 1 is a pre-Copernican view or our Solar System. It was found that $8 \%$ used this model when talking about our Solar System. Of these students, $4 \%$ thought that the Earth was the centre of the Universe. Nearly all agreed that the order of bodies was Earth, Moon, Mercury, Venus, Sun, Jupiter etc. This framework has been dubbed Notion One.

Notion Two, shown in Figure 2, has all but the Earth in its correct position. All planets orbit the Sun except the Earth which is the centre of the Solar System and thus has the Sun orbiting it. The Moon also orbits the Earth and is inside the orbit of the Sun. $6 \%$ of students indicated this model.

Notion three (Fig. 3) finds the Sun being placed firmly in the centre of our Solar System. $8 \%$ of the students indicated that this was their framework with $40 \%$ of those thinking that the Sun was not the centre of the Universe. This is the first notion in which one student positively states that the Sun is moving in a direction away from the Big Bang.

One student $(<2 \%)$ indicated this model which is included as Notion four (Fig. 4) purely because it demonstrates the method of additions to a concept. Apart from the moon's orbit (dotted) the model is correct. On questioning, the student replied that the moon went round 


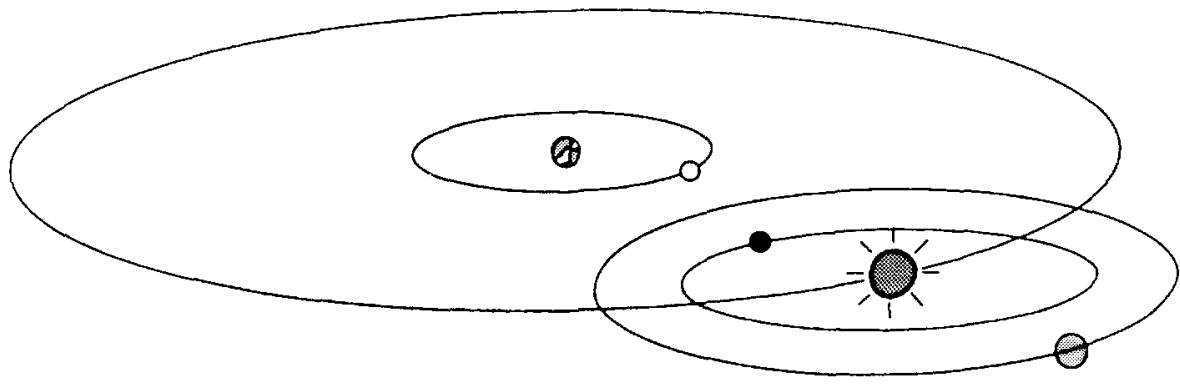

Figure 2.

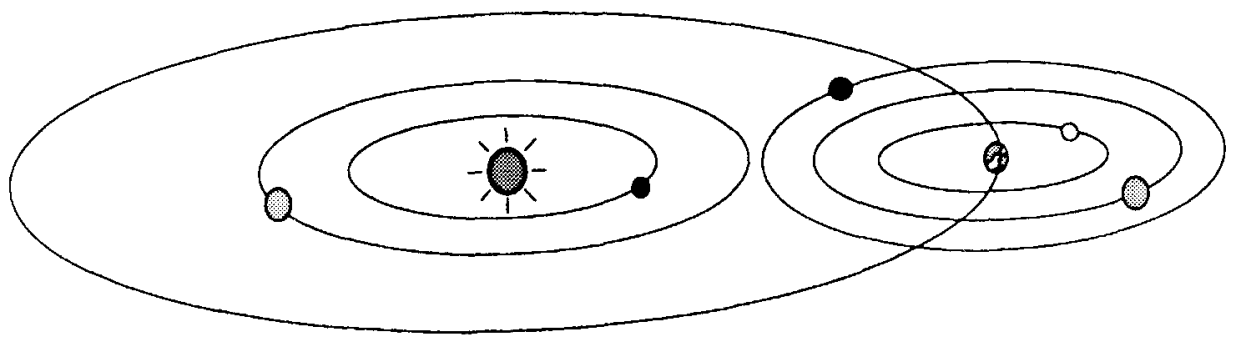

Figure 3.

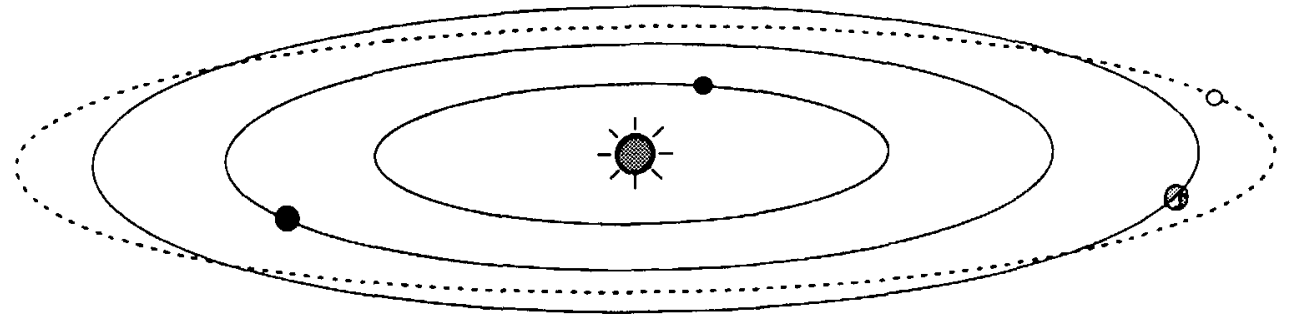

Figure 4. 


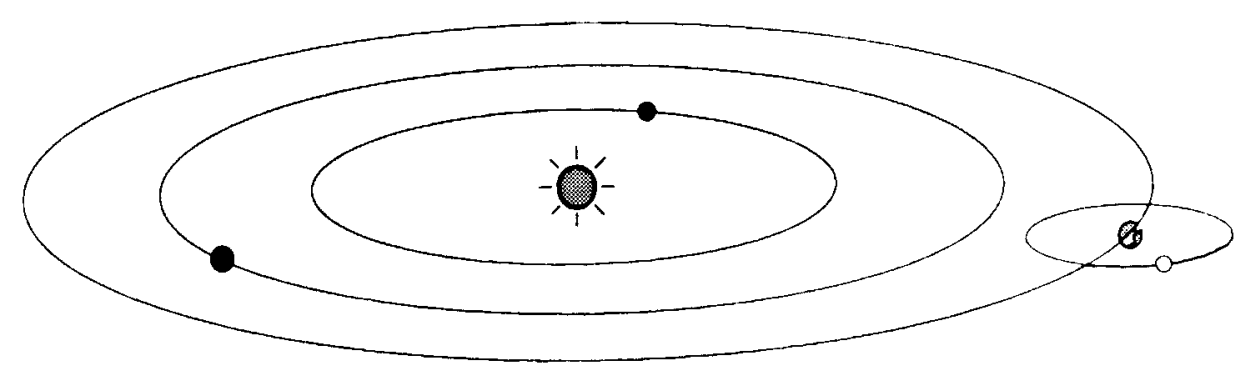

Figure 5.

the Sun, and its distance from the Sun made the phases. When asked to draw the model, the student drew the above picture very quickly and explained that the outer lobes are when the moon is 'growing' (waxing) and the inner when it is 'shrinking' (waning).

The correct model, notion five (Fig. 5), was chosen by the remaining $73 \%$ of the students. $11 \%$ of these students stated that the Sun moves in some manner, often away from the Big Bang. A further $28 \%$ said that they were almost certain that the Sun was static with the remainder either happy to abstain or not fall categorically either way.

\section{Further study}

The results found in this paper were slightly worse than expected. Further analysis and more students were thought necessary and so the Open University was approached. The Open University Astronomy course S281 is the largest single course in Europe (student numbers) and they kindly agreed to the University of Plymouth testing all the students prior to their taking the course. (S281 is a 2nd level programme).

After the University of Plymouth questionnaire, which is now seen as a pilot project, the last two questions were removed (the map questions on de-centering) and replaced with questions dealing with orbits of the Solar System and also on the wider universe. The questions asked both for movement and diagrams to be given. One phases question was also removed leaving two similar.

In total 1200 questionnaires were sent out to open University students, with the current number returned at 347. It is expected that not many more will be returned. Initial results are appearing to be very similar to the results gained at the University of Plymouth, and are listed below in Table 2.

Table 2

Question Overview

Correct phases response

Correct eclipse response

Correct Solar System drawn $\begin{array}{ll} & \text { Common Justifications for phases } \\ 78 \% & \text { Moon Blocks light } \\ 91 \% & \text { Earth casts a shadow } \\ 81 \% & \text { Moon becomes more reflective } \\ & \text { Moon moves away from Earth }\end{array}$ 


\section{Conclusion to the surveys}

The findings presented here are shown in a compacted form, yet still show the general trend of 'basic astronomical models' held by undergraduate astronomy students. Although no other research exists in this field, the data does follow from other researchers' findings such as Acker and Pecker (1990), who quizzed the general public, and also those of Durant (1989).

The research shows that phases are often able to be correctly positioned with respect to the Earth-Moon-Sun system, yet the underlying knowledge of the events is still incorrect.

Evidence of altering frameworks exists, allowing the student to fit new models to existing ideas allowing events, such as phases, to again 'work'.

The result of the full survey of Open University students is still being analysed but will soon be complete. Preliminary results of the survey and also those of the University of Plymouth survey suggest that Astronomy courses need to take account of the students' 'basic astronomical conceptions' before attempting to teach more advanced techniques.

If this is not adhered to, we may well find students applying relativity, albeit correctly, to Ptolemy's Universe.

\section{REFERENCES}

Acker, A. \& Pecker, J.C., 1990 Public misconceptions about Astronomy Cambridge, Cambridge University Press, IAU Colloquium no.105.

Berry, J. \& Graham, E., 1991, Using concept questions in teaching mechanics Int. J. Math. Educ. Sci. Technol., Vol 22, No 5, 749-757.

Durant, J.R. 1989, Public understanding of Science, Nature, Vol 340, 11-14.

Mali AND Howe, G.B. \& Howe, A., 1979, Development of Earth and Gravity concepts amoung Nepali Children, Science Edu, Vol 63, \#5, 685-691.

PIAGET, J., 1929, The Childs conception of the world, Sarreod and Sons Ltd, ISBN 0710030681.

Vosniadou, S.\& Brewer, W.F., 1989, Mental Models of the Earth, Cognitive Psychology, 035-564. 